\title{
Safety of Dienogest and Other Hormonal Treatments for Endometriosis in Real-World Clinical Practice (VIPOS): A Large Noninterventional Study
}

\author{
Klaas Heinemann · Bruno Imthurn · Lena Marions • Christoph Gerlinger • \\ Kerstin Becker · Sabine Moehner · Thomas Faustmann
}

Received: October 23, 2019 / Published online: April 16, 2020

(C) The Author(s) 2020

\begin{abstract}
Introduction: Endometriosis is a common gynecologic disease associated with a significant burden on women's health and healthcare systems. Currently approved hormonal treatments
\end{abstract}

Digital Features To view digital features for this article go to https://doi.org/10.6084/m9.figshare.12046359.

Electronic Supplementary Material The online version of this article (https://doi.org/10.1007/s12325$020-01331-z)$ contains supplementary material, which is available to authorized users.

K. Heinemann $(\bowtie) \cdot$ K. Becker $\cdot$ S. Moehner

ZEG Berlin, 10115 Berlin, Germany

e-mail: k.heinemann@zeg-berlin.de

B. Imthurn

Department of Reproductive Endocrinology,

University Hospital Zurich, 8091 Zurich,

Switzerland

L. Marions

Department of Clinical Science and Education,

Karolinska Institutet, 11883 Stockholm, Sweden

C. Gerlinger

Statistics and Data Insights, Bayer AG, 13553 Berlin,

Germany

C. Gerlinger

Gynecology, Obstetrics and Reproductive Medicine,

University Medical School of Saarland,

66421 Homburg, Saar, Germany

T. Faustmann

Bayer AG, 13353 Berlin, Germany for endometriosis can be effective in controlling symptoms, but may have clinically relevant side effects that limit their long-term use. Dienogest $2 \mathrm{mg}$ (Visanne; Bayer AG, Berlin, Germany) is a 19-nortestosterone derivative that significantly reduces menstrual bleeding, dysmenorrhea, premenstrual pain, dyspareunia, and pelvic pain in women with endometriosis. Although dienogest $2 \mathrm{mg}$ has demonstrated efficacy in clinical trials, data regarding long-term and realworld use are limited.

Methods: To our knowledge, the Visanne Postapproval Observational Study (VIPOS) is the largest real-world, noninterventional study performed examining the safety of dienogest and other hormonal treatments for the management of endometriosis in routine clinical practice. Patients self-reported medical and gynecologic history and symptoms and treatment information. Primary clinical outcomes were clinically validated and subject to independent blinded adjudication. Loss to follow-up was minimized through active contact with participating women at 6 months postenrollment and annually thereafter to ensure almost all clinically relevant outcomes were captured.

Planned Outcomes: VIPOS planned to enroll approximately 25,000 women initiating a new treatment for endometriosis, including those prescribed dienogest $2 \mathrm{mg} /$ day and other hormonal medications for endometriosis (approved or nonapproved), from approximately 
1000 centers in six European countries. The main clinical outcomes of interest for follow-up are anemia requiring medical intervention, de novo or clinically worsening depression, and treatment-failure patterns that result in drug discontinuation. Additional analyses will characterize the baseline risk factors of medically managed patients with endometriosis and assess treatment utilization patterns. VIPOS was designed to provide real-world information on endometriosis treatment and associated clinical outcomes, while not affecting the prescribing physician's decisions or the classification of patient diagnoses.

Trial Registration: European Union Electronic Register of Post-Authorisation Studies (EU PAS) no. 1613, Clinicaltrials.gov: NCT01266421.

Keywords: Dienogest; Endometriosis; Hormonal treatment; Observational; Prospective; Realworld evidence; Women's health

\section{Key Summary Points}

To our knowledge, the Visanne Postapproval Observational Study (VIPOS) is the largest real-world, non-interventional study evaluating the medical management of endometriosis, and we believe that data from VIPOS can provide insights into current treatment practices for endometriosis in Europe, the women who typically present to their healthcare providers, and their journey as a patient with endometriosis.

VIPOS is supported by a robust study design, characterized by sensitive and regular data collection, and uses multiple validation steps, which aim to minimize bias and misclassification of patientreported clinical outcomes.

The study design of VIPOS should minimize loss to follow-up through direct contact with the women enrolled, thereby ensuring that the impact of different longterm hormonal treatments on clinical outcomes across distinct healthcare settings can be accurately assessed.
VIPOS assesses a large, diverse population of women with endometriosis, which we believe is valuable to evaluate women's and physician's behavior, rare events, and subtle safety signals associated with endometriosis treatments.

\section{INTRODUCTION}

Endometriosis is a chronic, estrogen-dependent disease that affects $10-15 \%$ of women during their reproductive years and is defined by the presence of endometrial-like tissue outside the uterus cavity that induces chronic inflammation, ovarian cyst formation, and fibrosis [1, 2]. While the pathogenesis of endometriosis is unclear, inflammation is now accepted as central in the development and progression of the disease, characterized by an overproduction of inflammatory mediators, such as prostaglandins, metalloproteinases, cytokines, and chemokines [3]. In fact, investigation of the expression of inflammatory cytokines in peritoneal fluid of women with endometriosis has suggested that interleukin (IL)-17A and IL-2 are involved in inflammatory processes underlying endometriosis [4]. Moreover, reactive oxygen species and free radicals may lead to the growth and adhesion of endometrial cells in the peritoneal cavity and thereby disease onset [3].

The symptoms of endometriosis can include pain, bleeding disorders, and impaired fertility and represent a significant burden on women's health and healthcare systems worldwide [1]. Furthermore, endometriosis is known to compromise women's social relationships, sexuality, and psychologic and mental health $[5,6]$.

The number of licensed pharmaceutical agents for the treatment of endometriosis is currently limited. Medical treatment of endometriosis-associated pain is based on suppression of estrogen production and induction of amenorrhea [7], and treatments are often accompanied by clinically relevant side effects [8]. Gonadotropin-releasing hormone (GnRH) agonists are an example; they are generally only prescribed for 3-6 months at a time because of symptoms of estrogen deprivation, 
including vaginal dryness and hot flushes, and their negative impact on bone mineral density $[1,9]$. Likewise, the testosterone analog danazol is no longer broadly prescribed for endometriosis, in particular because of its androgenizing side effects [9]. Danazol has been removed from many markets but is still prescribed in some European countries, including Poland, Ukraine, and Russia. Consequently, there is an unmet need for medical therapies that are suitable for the longterm management of endometriosis.

Dienogest is a 19-nortestosterone derivative progestogen that is highly selective for progesterone receptors, while demonstrating only negligible binding for estrogen, androgen, glucocorticoid, and mineralocorticoid receptors $[10,11]$. Furthermore, dienogest does not cause metabolic imbalance, and treatment with dienogest can be prescribed as a continuous regimen [11]. Evidence has supported the comparable efficacy of dienogest with GnRH agonists in controlling endometriosisassociated pain symptoms [12]. In 2010, dienogest $2 \mathrm{mg}$ was approved in Europe for the treatment of endometriosis after demonstrating efficacy in patients with endometriosis by improving dysmenorrhea, premenstrual pain, dyspareunia, and diffuse endometriosisassociated pelvic pain as well as decreasing the duration of menstrual bleeding and the size of endometriomas $[9,13-16]$. Since then, dienogest $2 \mathrm{mg}$ has been further investigated in heterogeneous populations, in which the initial observations regarding efficacy and safety have been confirmed for the long-term treatment of endometriosis $[9,13-15,17,18]$.

Nevertheless, some safety and tolerability concerns exist over the role of progestins in treating endometriosis, particularly surrounding mood disturbances, depressive symptoms, and bleeding disturbances [19-21].

Real-world observational studies are gaining credibility as a study genre, serving as an important adjunct to randomized controlled clinical trials by addressing clinically relevant questions that cannot be answered within a controlled setting [22]. To date, economic, clinical, and patient-reported outcomes have been investigated in the field of endometriosis, including patient and disease characteristics in clinical practice, treatment adherence, impact of simultaneous treatments, and trends in incidence and disease management [22-25]. In addition, real-world studies have indicated the role that cultural influence, specialist access, healthcare shortcomings, and socioeconomic status have in the well-established diagnostic delay in endometriosis $[24,26,27]$. Prior to this study, there had been minimal investigation into the real-world management of endometriosis, and cohorts recruited have often involved small numbers of women at single centers with limited follow-up periods $[28,29]$.

Here we report the design of the Visanne (dienogest) Post-approval Observational Study (VIPOS), a noninterventional post-authorization safety study of dienogest in a real-world setting. VIPOS assesses the real-world safety of dienogest and other hormonal treatments administered, characterizes the baseline demographics of medically managed patients with endometriosis, analyzes their treatment utilization patterns, and investigates the risks associated with long-term medical treatment for women with endometriosis. To our knowledge, VIPOS is the largest observational study evaluating the real-world management of patients with endometriosis and provides a unique opportunity to expand our understanding of endometriosis and its management across Europe.

\section{METHODS}

\section{Study Design}

VIPOS is a prospective, noninterventional, long-term active surveillance cohort study of women using various hormonal treatments for endometriosis across six European countries: Germany, Hungary, Poland, Russia, Switzerland, and Ukraine. The study was a postmarketing authorization measure after European approval and incorporated similar methodology to the European/International Active Surveillance study, which previously established the standard for evaluation of post-marketing authorization safety studies for hormonal drug treatments [30, 31]. 
Overall, approximately 25,000 women with endometriosis who were initiating a new treatment regimen were planned for enrollment at approximately 1000 study centers [32]. The enrolling physician provided details of the newly prescribed treatment for endometriosis and the diagnostic classification (clinical or surgical confirmation; Fig. 1). The purpose of the "noninterference" approach of the study was to provide standardized, comprehensive, and reliable information on endometriosis treatment patterns. In addition, study assessments were not intended to interfere with the prescribing behavior of physicians or with the individual needs of the participating women.

\section{Sample Selection}

All women initiating a new treatment regimen for endometriosis (i.e., first-time users or treatment switchers) and who were willing to take part in the long-term observational study were eligible for inclusion. This recruitment approach ensured that the study cohort was representative of real-world users of each prescribed endometriosis treatment. There were no specific medical inclusion or exclusion criteria, but women with a language barrier or who were not cooperative/available for follow-up were not eligible to participate. Women who discontinued endometriosis treatment during the study continued to be followed up, provided they had not withdrawn study consent.

VIPOS was initiated in Germany and Poland in late 2010 and in Hungary in early 2011. Due to recruitment challenges, Ukraine, Russia, and Switzerland were included to broaden the recruitment base, with recruitment starting in late 2011 in Russia and Ukraine and in 2012 for Switzerland. Women participating in the study were followed for up to 7 years, and the followup period ended in late 2018 [32].

\section{Measurements}

At baseline, medical and gynecologic history and endometriosis-related symptoms were directly self-reported by women with endometriosis, and diagnosis and treatment were reported by physicians via extensive questionnaires. The additional files include these detailed questionnaires (Additional Files S1 and S2). Each patient was actively followed up every 6 months for the first year of treatment, then annually from the second year until the end of the study (up to 7 years after study enrollment), to ensure all relevant adverse events were captured.

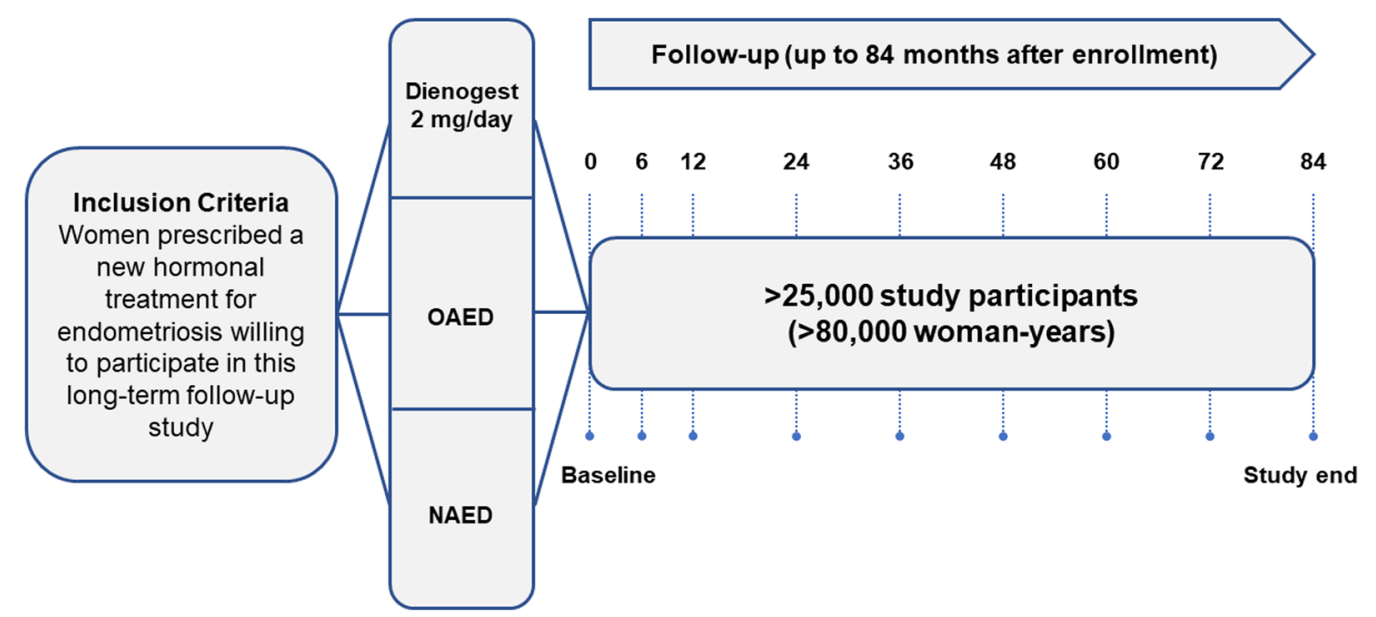

Fig. 1 VIPOS study design. NAED nonapproved hormonal medications for endometriosis, OAED other hormonal medications approved for endometriosis 


\section{PLANNED OUTCOMES}

The primary study objectives were to assess the safety of oral dienogest $2 \mathrm{mg}$ /day compared with other hormonal treatments for endometriosis in routine clinical practice. Three primary safety endpoints of interest were anemia requiring medical intervention, de novo or clinically worsening depression, and drug discontinuation due to treatment failure (Table 1). Secondary study objectives included the characterization of baseline risk factors (lifetime history of comorbidity, co-medication, risk markers, sociodemographic data, and lifestyle data) and the analysis of drug utilization patterns. While this study was primarily developed to evaluate the safety of dienogest for

Table 1 Summary of study objectives

\begin{tabular}{lc}
\hline $\begin{array}{l}\text { Clinical outcomes of } \\
\text { interest for primary } \\
\text { objective }\end{array}$ & Secondary objectives \\
\hline $\begin{array}{l}\text { Occurrence of anemia } \\
\text { induced by cyclical }\end{array}$ & $\begin{array}{c}\text { Characterize baseline risk } \\
\text { factors of real-world }\end{array}$ \\
$\begin{array}{l}\text { bleeding disturbances } \\
\text { - Noninferiority test for }\end{array}$ & $\begin{array}{l}\text { patients (sociodemographic } \\
\text { and lifestyle data, risk }\end{array}$ \\
$\begin{array}{l}\text { dienogest } 2 \text { mg/day vs. } \\
\text { other hormonal } \\
\text { treatments }\end{array}$ & $\begin{array}{l}\text { co-medication) } \\
\text { Newly reported or } \\
\text { worsening clinically } \\
\text { relevant depression }\end{array}$ \\
$\begin{array}{l}\text { - Noninferiority test for } \\
\text { dienogest } 2 \text { mg/day vs. } \\
\text { other hormonal } \\
\text { treatments }\end{array}$ & $\begin{array}{l}\text { Analyze drug-use patterns of } \\
\text { endometriosis treatments in } \\
\text { Discontinuation due to } \\
\text { treatment failure } \\
\text { dienogest } 2 \text { mg/day vs. } \\
\text { other hormonal } \\
\text { treatments }\end{array}$ \\
\hline
\end{tabular}

the treatment of endometriosis, these additional endpoints provide further clinical insight from a large data set encompassing data on the real-world symptoms, diagnosis, and treatment of endometriosis in Europe.

Overall, the study outcomes in VIPOS are expected to reveal information about current treatment practices in Europe, the women who typically present to their health services, and the journey of a patient with endometriosis. The insights gained from these outcomes have the potential to translate into better-informed treatment practices, with a greater understanding of the efficacy and safety of the medical management of endometriosis.

\section{Data Collection}

To minimize bias and misclassification of patient-reported clinical outcomes, multiple validation steps were applied. All relevant clinical events (i.e., anemia and depression) were reported during follow-up via a questionnaire and sent to the clinical research organization, ZEG Berlin (Berlin Center for Epidemiology and Health Research), which contacted the relevant physician and patient, as needed, to confirm the event. Missing or incomplete data for most relevant items were clarified by contacting the patient. In addition, all reported events of anemia and depression were categorized, using a predefined validated algorithm, as "confirmed" or "not confirmed." Finally, verification of these cases at the end of study was undertaken by two blinded independent medical boards appointed by the Safety Monitoring and Advisory Council. The Hematology Board consisted of three independent medical experts who specialize in internal medicine/hematology and gynecology, while the Depressive Illness Board consisted of specialists in psychiatry and psychologic medicine.

\section{Data Analysis}

A noninferiority study design was chosen based on the a priori assumption that use of dienogest is not associated with an increased risk of anemia or depression compared with other 
approved hormonal medications. Three hypotheses were to be tested; therefore, Bonferroni-Holm correction was used to maintain the overall error rate by testing each individual hypothesis based on an $\alpha$ level of 0.0167 (a statistical significance level of one third of that required if only one hypothesis were to be tested).

The study was powered to exclude a two-fold risk of anemia and clinically relevant depression with dienogest $2 \mathrm{mg}$ compared with other endometriosis medications, based on the expected incidence of 1 event per 100 womanyears, with a noninferiority margin of 0.01 and $90 \%$ power. Additionally, VIPOS was powered to demonstrate the superiority of dienogest $2 \mathrm{mg}$ compared with other endometriosis medications in relation to treatment failure, with a clinically relevant difference of 0.05 , and 90\% power.

Power calculations based on the expected incidences of anemia and depression indicated approximately 84,000 women-years as sufficient to demonstrate non-inferiority of dienogest compared with other endometriosis medications for anemia and depression. It was calculated that approximately 29,500 women-years would be required to demonstrate the superiority of dienogest compared with other endometriosis medications regarding treatment failure. The original study size was based on the 3-6-year follow-up of 25,000 women, which would provide approximately 89,000 documented womanyears, assuming a $10 \%$ dropout rate and 3 years to complete recruitment.

\section{STRENGTHS AND LIMITATIONS}

The VIPOS post-authorization safety study is the largest real-world, noninterventional evaluation of the medical management of endometriosis and is expected to provide important data regarding the long-term safety and efficacy of dienogest and other hormonal treatments for endometriosis in clinical practice. Previous real-world data sets used to investigate the medical treatment of endometriosis have used substantially smaller cohorts, often derived from single centers, with a comparatively short follow-up period [28, 29]. By following patients for a minimum of 3 years and for up to 7 years, VIPOS will also provide greater insight into the impact of patient baseline characteristics on the real-world treatment journey for patients with endometriosis. VIPOS will assess a large and diverse population so that rare events and subtle safety signals associated with endometriosis treatments may also be investigated, alongside patterns of patient adherence and physician behavior.

Although there may be differences between the VIPOS cohorts and other populations of women with endometriosis internationally, the study is also expected to identify trends in clinical practice within countries, such as treatment utilization and diagnostic methods, as well as any regional differences in diagnostic tools and therapy. It will also provide a description of a large cohort of women with endometriosis in routine clinical practice. As such, VIPOS will expand our understanding of long-term hormonal therapy, treatment adherence risks, and impact on patient quality of life for women with endometriosis.

A potential limitation of previous real-world observational studies of endometriosis is the threat to data validity from measurement error, unmeasured confounding, missing data, diagnostic criteria, and selection bias $[28,29,33-35]$. The robust study design of VIPOS aims to minimize these risks through sensitive and regular collection of data using multiple validation steps, including verification of reported anemia and depression by blinded independent adjudication. In addition, the study design should minimize loss to follow-up through direct contact with the women enrolled. As a result, the impact of different long-term hormonal treatments on clinical outcomes across distinct healthcare settings can be accurately assessed. In addition, the complexities and potential interaction among depression, endometriosis, and progestogens have previously made it difficult to differentiate whether an individual's depressive symptoms are causally associated with progestin use or with sequelae of the disease process. To this end, VIPOS is needed to evaluate the influence of dienogest $2 \mathrm{mg}$ on long-term safety in 
women with endometriosis, including mood disturbances and depression, and to compare these outcomes with those observed for other hormonal treatments.

\section{ETHICS AND DISSEMINATION}

The VIPOS study was approved by one independent ethics committee/institutional review board at each country, where required. In Germany, the Ethics Committee of the Berlin Medical Association approved the study, and in Hungary this was done by the Scientific and Research Ethics Committee of the Medical Research Council. In Switzerland, only one large endometriosis center (Inselspital Bern) took part in the study, and a positive vote from Swissmedic was obtained for this hospital. No ethical approval for non-interventional, observational studies was required by law in Poland, Russia, and Ukraine. Each woman was asked to provide written informed consent before participating in the study. The study was conducted in accordance with the Guidelines for Good Pharmacoepidemiology Practices issued by the International Society for Pharmacoepidemiology (2008), the Good Epidemiological Practice-Proper Conduct in Epidemiologic Research statement issued by the International Epidemiological Association European Federation (2007), the European Network of Centres for Pharmacoepidemiology and Pharmacovigilance (ENCePP) Code of Conduct for Scientific Independence and Transparency (2010), and the ethical principles based on the Declaration of Helsinki. The study was prospectively registered in the EU PAS as number 1613 on October 21, 2010, and received an ENCePP seal. In addition, this study was registered on CinicalTrials.gov (NCT01266421) on December 24, 2010.

\section{ACKNOWLEDGEMENTS}

Funding. The VIPOS study was independently run by ZEG Berlin, funded by an unconditional grant from Bayer Pharmaceuticals (Berlin,
Germany) and supervised by an independent Safety Monitoring and Advisory Council. However, Bayer (respectively Schering at that time) had peer reviewed the study protocol. All authors had full access to all of the data in this study and take complete responsibility for the integrity of the data and the accuracy of its analysis. The Rapid Service and Open Access Fees for the journal were funded by Bayer AG.

Medical Writing Assistance. Medical writing support was provided by Afsaneh Khetrapal (ApotheCom, London, UK) and funded by Bayer AG.

Authorship. All named authors meet the International Committee of Medical Journal Editors (ICMJE) criteria for authorship for this article, take responsibility for the integrity of the work as a whole, and have given their approval for this version to be published.

Disclosures. Klaas Heinemann, Kerstin Becker, and Sabine Moehner are full-time employees at ZEG Berlin. Bruno Imthurn has nothing to disclose. Lena Marions is a principal investigator for trials sponsored by Bayer AG and Merck Sharp \& Dohme. Christoph Gerlinger and Thomas Faustmann are full-time employees at Bayer AG.

Compliance with Ethics Guidelines. The VIPOS study was approved by one independent ethics committee/institutional review board at each country, where required. In Germany, the Ethics Committee of the Berlin Medical Association approved the study, and in Hungary this was done by the Scientific and Research Ethics Committee of the Medical Research Council. In Switzerland, only one large endometriosis center (Inselspital Bern) took part in the study, and a positive vote from Swissmedic was obtained for this hospital. No ethical approval for non-interventional, observational studies was required by law in Poland, Russia, and Ukraine. Each woman was asked to provide written informed consent before participating in the study. The study was conducted in accordance with the Guidelines for Good Pharmacoepidemiology Practices issued 
by the International Society for Pharmacoepidemiology (2008), the Good Epidemiological Practice-Proper Conduct in Epidemiologic Research statement issued by the International Epidemiological Association European Federation (2007), the European Network of Centres for Pharmacoepidemiology and Pharmacovigilance (ENCePP) Code of Conduct for Scientific Independence and Transparency (2010), and the ethical principles based on the Declaration of Helsinki. The study was prospectively registered in the EU PAS as number 1613 on October 21, 2010, and received an ENCePP seal. In addition, this study was registered on CinicalTrials.gov (NCT01266421) on December 24, 2010.

Data Availability. This manuscript has no associated data or the data will not be deposited.

Open Access. This article is licensed under a Creative Commons Attribution-NonCommercial 4.0 International License, which permits any non-commercial use, sharing, adaptation, distribution and reproduction in any medium or format, as long as you give appropriate credit to the original author(s) and the source, provide a link to the Creative Commons licence, and indicate if changes were made. The images or other third party material in this article are included in the article's Creative Commons licence, unless indicated otherwise in a credit line to the material. If material is not included in the article's Creative Commons licence and your intended use is not permitted by statutory regulation or exceeds the permitted use, you will need to obtain permission directly from the copyright holder.To view a copy of this licence, visit http://creativecommons.org/licenses/by$\mathrm{nc} / 4.0 /$.

\section{REFERENCES}

1. Wills HDC, May K, Kennedy S, Kirtley S, Hogg S. NHS evidence-endometriosis annual evidence update. 2010.
2. Bulun SE, Cheng YH, Pavone ME, Xue Q, Attar E, Trukhacheva E, Tokunaga $\mathrm{H}$, Utsunomiya $\mathrm{H}$, Yin $\mathrm{P}$, Luo $\mathrm{X}$, et al. Estrogen receptor-beta, estrogen receptor-alpha, and progesterone resistance in endometriosis. Semin Reprod Med. 2010;28(1): 36-433.

3. Vitale SG, Capriglione S, Peterlunger I, La Rosa VL, Vitagliano A, Noventa M, Valenti G, Sapia F, Angioli R, Lopez S, et al. The role of oxidative stress and membrane transport systems during endometriosis: a fresh look at a busy corner. Oxid Med Cell Longev. 2018;2018:7924021.

4. Fan YY, Chen HY, Chen W, Liu YN, Fu Y, Wang LN. Expression of inflammatory cytokines in serum and peritoneal fluid from patients with different stages of endometriosis. Gynecol Endocrinol. 2018;34(6): 507-12.

5. Lagana AS, La Rosa VL, Rapisarda AMC, Valenti G, Sapia F, Chiofalo B, Rossetti D, Ban Frangez H, Vrtacnik Bokal E, Vitale SG. Anxiety and depression in patients with endometriosis: impact and management challenges. Int J Womens Health. 2017;9: 323-30.

6. Facchin F, Barbara G, Dridi D, Alberico D, Buggio L, Somigliana E, Saita E, Vercellini P. Mental health in women with endometriosis: searching for predictors of psychological distress. Hum Reprod. 2017;32(9):1855-61.

7. Bedaiwy MA, Alfaraj S, Yong P, Casper R. New developments in the medical treatment of endometriosis. Fertil Steril. 2017;107(3):555-65.

8. Dunselman GA, Vermeulen N, Becker C, CalhazJorge C, D'Hooghe T, De Bie B, Heikinheimo O, Horne AW, Kiesel L, Nap A, et al. ESHRE guideline: management of women with endometriosis. Hum Reprod. 2014;29(3):400-12.

9. Strowitzki T, Marr J, Gerlinger C, Faustmann T, Seitz C. Dienogest is as effective as leuprolide acetate in treating the painful symptoms of endometriosis: a 24-week, randomized, multicentre, open-label trial. Hum Reprod. 2010;25(3):633-41.

10. Mueck AO. Dienogest: an oral progestogen for the treatment of endometriosis. Expert Rev Obstet Gynecol. 2011;6(1):5-15.

11. Lagana AS, Vitale SG, Granese R, Palmara V, Ban Frangez H, Vrtacnik-Bokal E, Chiofalo B, Triolo O. Clinical dynamics of Dienogest for the treatment of endometriosis: from bench to bedside. Expert Opin Drug Metab Toxicol. 2017;13(6):593-6.

12. Andres Mde P, Lopes LA, Baracat EC, Podgaec S. Dienogest in the treatment of endometriosis: 
systematic review. Arch Gynecol Obstet. 2015;292(3):523-9.

13. Kohler G, Faustmann TA, Gerlinger C, Seitz C, Mueck AO. A dose-ranging study to determine the efficacy and safety of 1,2 , and $4 \mathrm{mg}$ of dienogest daily for endometriosis. Int $\mathrm{J}$ Gynaecol Obstet. 2010;108(1):21-5.

14. Petraglia F, Hornung D, Seitz C, Faustmann T, Gerlinger C, Luisi S, Lazzeri L, Strowitzki T. Reduced pelvic pain in women with endometriosis: efficacy of long-term dienogest treatment. Arch Gynecol Obstet. 2012;285(1):167-73.

15. Strowitzki T, Faustmann T, Gerlinger C, Seitz C. Dienogest in the treatment of endometriosis-associated pelvic pain: a 12 -week, randomized, doubleblind, placebo-controlled study. Eur J Obstet Gynecol Reprod Biol. 2010;151(2):193-8.

16. Bayer: Visanne-summary of product characteristics (SPC). https://db.cbg-meb.nl/mri/spc/nlh-1569001.pdf. Accessed 8 Oct 2018

17. Ebert AD, Dong L, Merz M, Kirsch B, Francuski M, Bottcher B, Roman H, Suvitie P, Hlavackova O, Gude K, et al. Dienogest $2 \mathrm{mg}$ daily in the treatment of adolescents with clinically suspected endometriosis: The VISanne Study to Assess Safety in ADOlescents. J Pediatr Adolesc Gynecol. 2017;30(5):560-7.

18. Lang J, Yu Q, Zhang S, Li H, Gude K, von Ludwig C, Ren X, Dong L. Dienogest for treatment of endometriosis in Chinese women: a placebo-controlled, randomized, double-blind phase 3 study. J Womens Health (Larchmt). 2018;27(2):148-55.

19. Harada T, Taniguchi F. Dienogest: a new therapeutic agent for the treatment of endometriosis. Womens Health (Lond). 2010;6(1):27-35.

20. Panay N, Studd J. Progestogen intolerance and compliance with hormone replacement therapy in menopausal women. Hum Reprod Update. 1997;3(2):159-71.

21. Sugimoto K, Nagata C, Hayashi H, Yanagida S, Okamoto A. Use of dienogest over 53 weeks for the treatment of endometriosis. J Obstet Gynaecol Res. 2015;41(12):1921-6.

22. Heikinheimo O, Bitzer J, Garcia Rodriguez L. Realworld research and the role of observational data in the field of gynaecology-a practical review. Eur J Contracept Reprod Health Care. 2017;22(4):250-9.

23. Dreyer NA, Tunis SR, Berger M, Ollendorf D, Mattox P, Gliklich R. Why observational studies should be among the tools used in comparative effectiveness research. Health Aff (Millwood). 2010;29(10): 1818-25.

24. Matalliotakis M, Goulielmos GN, Matalliotaki C, Trivli A, Matalliotakis I, Arici A. Endometriosis in adolescent and young girls: report on a series of 55 cases. J Pediatr Adolesc Gynecol. 2017;30(5): 568-70.

25. Saavalainen L, Tikka T, But A, Gissler M, Haukka J, Tiitinen A, Harkki P, Heikinheimo O. Trends in the incidence rate, type and treatment of surgically verified endometriosis-a nationwide cohort study. Acta Obstet Gynecol Scand. 2018;97(1):59-67.

26. Eisenberg VH, Weil C, Chodick G, Shalev V. Epidemiology of endometriosis: a large populationbased database study from a healthcare provider with 2 million members. BJOG. 2018;125(1):55-62.

27. Morassutto C, Monasta L, Ricci G, Barbone F, Ronfani L. Incidence and estimated prevalence of endometriosis and adenomyosis in Northeast Italy: a data linkage study. PLoS ONE. 2016;11(4): e0154227.

28. Maiorana A, Incandela D, Parazzini F, Alio W, Mercurio A, Giambanco L, Alio L. Efficacy of dienogest in improving pain in women with endometriosis: a 12-month single-center experience. Arch Gynecol Obstet. 2017;296(3):429-33.

29. Park SY, Kim SH, Chae HD, Kim CH, Kang BM. Efficacy and safety of dienogest in patients with endometriosis: a single-center observational study over 12 months. Clin Exp Reprod Med. 2016;43(4): 215-20.

30. Kang JL, Wang XX, Nie ML, Huang XH. Efficacy of gonadotropin-releasing hormone agonist and an extended-interval dosing regimen in the treatment of patients with adenomyosis and endometriosis. Gynecol Obstet Invest. 2010;69(2):73-7.

31. Porpora MG, Brunelli R, Costa G, Imperiale L, Krasnowska EK, Lundeberg T, Nofroni I, Piccioni MG, Pittaluga E, Ticino A, et al. A promise in the treatment of endometriosis: an observational cohort study on ovarian endometrioma reduction by $N$-acetylcysteine. Evid Based Complement Alternat Med. 2013;2013:240702.

32. Heinemann K, Imthurn B, Marions L, Faustmann T. VIPOS: a large non-interventional study examining the safety of dienogest and other hormonal treatments for endometriosis in real-world clinical practice. The Society of Endometriosis and Uterine Disorders, 25-28 April, 2018, Florence, Italy.

33. Berger ML, Sox H, Willke RJ, Brixner DL, Eichler HG, Goettsch W, Madigan D, Makady A, Schneeweiss S, Tarricone $\mathrm{R}$, et al. Good practices for 
real-world data studies of treatment and/or comparative effectiveness: recommendations from the joint ISPOR-ISPE Special Task Force on real-world evidence in health care decision making. Pharmacoepidemiol Drug Saf. 2017;26(9):1033-9.

34. Dinger JC, Bardenheuer K, Assmann A. International active surveillance study of women taking oral contraceptives (INAS-OC study). BMC Med Res Methodol. 2009;9:77.

35. ZEG Berlin: Berlin Center for Epidemiology and Health Research: Post-authorization safety studies. https://zeg-berlin.de/expertise/post-authorizationsafety-studies/. Accessed 8 Oct 2018 